\title{
Análise da Revisão Cochrane: Administração Suplementar de Vitamina D para Prevenção de Cancro em Adultos. Cochrane Database Syst Rev. 2014, 6:CD007469.
}

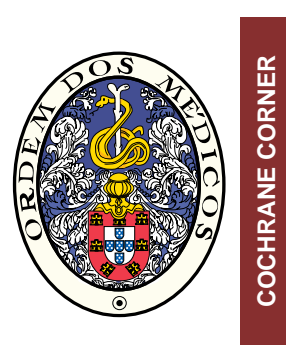

\author{
Analysis of the Cochrane Review: Vitamin D Supplementation for Prevention \\ of Cancer in Adults. Cochrane Database Syst Rev. 2014, 6:CD007469.
}

\author{
André Torres CARDOSO ${ }^{1,2}$, Liliana NANJI ${ }^{1,2}$, João COSTA ${ }^{1,2,3}$, António VAZ-CARNEIRO ${ }^{1,2}$ \\ Acta Med Port 2014 Jul-Aug;27(4):411-413
}

\begin{abstract}
RESUMO
A vitamina $D$ tem sido referida na literatura como sendo um agente potencialmente importante na prevenção de condições neoplásicas, incluindo cancro da mama, cólon, próstata e ovários. Contudo, a evidência científica actualmente disponível sobre o assunto é contraditória e inconclusiva. Na presente revisão sistemática Cochrane compararam-se indivíduos a fazer suplementação de vitamina D nas suas variadas formas (colecalciferol, ergocalciferol, alfacalcidol ou calcitriol), independentemente da dose, via ou duração de administração versus placebo, adultos sem qualquer intervenção ou adultos com uma doença em fase estável, não relacionada com o metabolismo da vitamina $D$. Os resultados indicam que actualmente não existe evidência consistente a favor de os suplementos de vitamina $D$ aumentarem ou diminuírem o risco de aparecimento de cancro, especialmente em mulheres idosas residentes na comunidade. Embora susceptíveis de terem sofrido erros de análise do tipo I por presença de RCTs com amostras reduzidas e por taxas de desistência significativas durante os estudos, a administração de suplementos de colecalciferol esteve associada a uma diminuição de 12\% (IC95\%: 2 a 22\%) do risco de mortalidade por cancro, tendo a administração de suplementos de vitamina D diminuído em $7 \%$ (IC95\%: 2 a 12\%) o risco de mortalidade global (por todas as causas). A administração combinada de suplementos de colecalciferol e cálcio aumentou a incidência de casos de nefrolitíase.
\end{abstract}

Palavras-chave: Adulto; Ensaios Clínicos Controlados Aleatórios como Assunto; Neoplasias/prevenção e controle; Revisão Sistemática; Vitamina D.

\section{ABSTRACT}

Vitamin D has been mentioned in the literature has a potentially important agent for preventing the development of tumors, namely breast, colon, prostate and ovary tumors. However, the currently available evidence on the subject is contradictory and inconclusive. In this Cochrane systematic review, patients taking supplemental vitamin D on its various forms (cholecalciferol, ergocalciferol, alfacalcidol or calcitriol), regardless the dose, duration and route of administration, were compared with placebo, healthy adults without any intervention or adults with a disease in a stable phase, non-related with vitamin D metabolism. The results showed that currently, there is no firm evidence that vitamin $D$ supplementation increases or decreases the risk of cancer occurrence, mainly in elderly community-dwelling women. Though at risk of type I errors due to small samples and substantial dropout of participants during the trials, the administration of supplemental cholecalciferol led to a $12 \%(\mathrm{Cl} 95 \%$ : 2 a $22 \%)$ decreased in cancer mortality, while the administration of supplemental vitamin D decreased all-cause mortality by $7 \%$ (Cl 95\%: 2 a 12\%). The combined administration of supplements of cholecalciferol and calcium induced an increased incidence of nephrolithiasis.

Keywords: Adult; Randomized Controlled Trials as Topic; Neoplasms/prevention and control; Systematic Reviews; Vitamin D.

\section{QUESTÃO CLÍNICA}

Os suplementos de vitamina $\mathrm{D}$ ajudam a prevenir o aparecimento de cancro?

\section{OBJETIVOS}

Esta revisão sistemática procurou avaliar os potenciais efeitos benéficos e prejudiciais da suplementação de vitamina $D$, na prevenção de aparecimento de cancro em indivíduos adultos.

\section{CRITÉRIOS DE SELECÇÃO}

Ensaios clínicos aleatorizados e controlados (RCTs) comparando indivíduos a fazer suplementação de vitamina D em qualquer dose, via ou duração de administração ver- sus placebo, adultos sem qualquer intervenção ou adultos com uma doença em fase estável, não relacionada com o metabolismo da vitamina D. A vitamina D foi administrada como monoterapia ou em associação com cálcio. Os suplementos de vitamina $D$ foram administrados sob a forma de colecalciferol, ergocalciferol, alfacalcidol ou calcitriol.

\section{METODOLOGIA}

Foram pesquisadas várias bases de dados - Cochrane Central Register of Controlled Trials (CENTRAL), (The Cochrane Library, Fevereiro 2014), Medline (até Fevereiro 2014), EMBASE (até Fevereiro 2014), LILACS (até Fevereiro 2014), Science Citation Index Expanded (até Feverei-

\footnotetext{
1. Centro de Estudos de Medicina Baseada na Evidência. Faculdade de Medicina. Universidade de Lisboa. Lisboa. Portugal.

2. Centro Colaborador Português da Rede Cochrane Iberoamericana. Lisboa. Portugal.

3. Unidade de Farmacologia Clínica. Instituto de Medicina Molecular. Faculdade de Medicina. Universidade de Lisboa. Lisboa. Portugal.

Recebido: 20 de Julho de 2014 - Aceite: 21 de Julho de 2014 | Copyright @ Ordem dos Médicos 2014
} 
ro 2014) - assim como registos de ensaios clínicos ainda em curso (www.clinicaltrials.gov/ e www.controlled-trials. com/ e a World Health Organization International Clinical Trials Registry Platform (ICTRP 2011)).

Foram ainda contactadas várias empresas farmacêuticas, a quem foram pedidos estudos aleatorizados ainda não publicados. A extracção dos dados e a avaliação do risco de vieses e de erros sistemáticos dos RCTs foi feita de forma independente por dois autores. Nas situações em que persistiam dúvidas, um terceiro elemento foi utilizado.

\section{RESULTADOS}

Foram incluídos nesta revisão sistemática $18 \mathrm{RCTs}$ (2 RCTs com baixo risco de vieses e 16 RCTs com risco incerto de vieses em pelo menos 1 dos 8 critérios utilizados pelos autores). Dos 18 RCTs, 16 foram financiados pela indústria farmacêutica. A totalidade dos estudos foi conduzida em países de rendimento elevado, na Europa, América do Norte e Oceânia.

$\mathrm{Na}$ totalidade dos 18 RCTs foram estudados 50623 indivíduos (o número de participantes por RCT variou entre 70 e 36.282 indivíduos - mediana 313). A idade média dos participantes foi 69 anos (intervalo: 47 - 97 anos de idade) sendo $81 \%$ destes do sexo feminino. O período médio de administração de suplementos de vitamina $D$ foi de seis anos. 14 RCTs testaram suplementos de colecalciferol, três RCTs testaram suplementos de calcitriol e um RCT testou suplementos de ergocalciferol.

O resultado da comparação entre os grupos suplementos de vitamina $D$ versus controlo não mostrou diferenças significativas no risco de incidência de cancro (risk ratios [RR]: 1,00; IC 95\%: 0,94 a 1,06). Não se verificou heterogeneidade estatística $\left(I^{2}=0 \%\right)$ entre os resultados dos estudos. Não se observaram também diferenças significativas entre os seguintes subgrupos:

1. RCTs de baixo risco de viés versus elevado risco de viés;

2. RCTs que avaliaram a prevenção primária versus prevenção secundária;

3. RCTs com doses de suplementos de vitamina D inferiores a $20 \mathrm{ng} / \mathrm{mL}$ versus doses de suplementos de vitamina $D$ iguais ou superiores a $20 \mathrm{ng} / \mathrm{mL}$;

4. RCTs que utilizam suplementos de vitamina D e cálcio concomitantemente versus suplementos de vitamina D sem cálcio concomitante.

Por outro lado, o risco de mortalidade por cancro foi inferior nos doentes a fazer suplementos de vitamina $D$ comparativamente ao grupo de controlo (RR: 0,88; IC 95\%: 0,78 a 0,$98 ; I^{2}=0 \%$ ). Similarmente, o risco de morte em geral, por todas as causas, também foi inferior nos doentes a fazer suplementos de vitamina D (RR: 0,93; IC 95\%: 0,88 a 0,$98 ; I^{2}=0 \%$ ). Nos indivíduos em que o cálcio foi associado a suplementos de vitamina $D$ verificou-se um risco acrescido de nefrolitíase comparativamente àqueles indivíduos apenas a fazer vitamina D (RR: 1,17; IC 95\%: 1,03 a 1,34; $\left.\mathrm{I}^{2}=0 \%\right)$. Em todos os casos a trial sequential analysis
(TSA) indicou que os achados acima poderiam dever-se a erros aleatórios.

\section{CONCLUSÕES}

Actualmente não existe evidência consistente a favor de os suplementos de vitamina D aumentarem ou diminuírem o risco de incidência de cancro, especialmente em mulheres idosas residentes na comunidade. Embora susceptíveis de terem sofrido erros de análise do tipo I por presença de RCTs com amostras reduzidas e por taxas de desistência significativas durante os estudos, a administração de colecalciferol induziu uma diminuição da mortalidade por cancro e a administração de vitamina $\mathrm{D}$ diminuiu a mortalidade em geral, para todas as causas. Também a administração combinada de suplementos de colecalciferol e cálcio aumentou a incidência de casos de nefrolitíase comparativamente à administração isolada de colecalciferol.

\section{COMENTÁRIO}

A vitamina $D$ tem uma grande diversidade de funções conhecidas, desde a homeostase do cálcio, à regulação de iões como o magnésio e o fósforo, à secreção hormonal pela $\mathrm{PTH}$, hipófise e pâncreas entre outras funções no sistema cardiovascular, muscular e imunitário. ${ }^{1}$ Contudo, algumas das funções que lhe são atribuídas não se encontram ainda devidamente comprovadas, nomeadamente a sua pretensa acção preventiva no aparecimento de situações neoplásicas.

A presente revisão sistemática teve por base uma série de RCTs em que foram comparados dois grupos de indivíduos:

- Um primeiro a realizar suplementação de vitamina $\mathrm{D}$;

- Um segundo correspondente ao grupo controlo em que não é feita a suplementação de vitamina $D$.

O objectivo foi compreender até que ponto a administração de suplementos de vitamina $\mathrm{D}$ poderá ser prejudicial ou benéfica na prevenção do aparecimento de cancro em adultos.

Os resultados globais reforçam a ideia de que actualmente não existe evidência consistente a favor de os suplementos de vitamina $D$ aumentarem ou diminuírem o risco de aparecimento de cancro, especialmente em mulheres idosas residentes na comunidade, população melhor representada nesta revisão sistemática. Simultaneamente a administração de colecalciferol induziu uma diminuição da mortalidade por cancro enquanto a administração de vitamina $\mathrm{D}$ diminuiu a mortalidade em geral, para todas as causas. Os intervalos de confiança destas estimativas são relativamente amplos, incluindo valores (por exemplo, $2 \%$ ) de difícil valorização clínica. Também a administração combinada de suplementos de colecalciferol e cálcio aumentaram a incidência de casos de nefrolitíase comparativamente à administração isolada de colecalciferol.

São necessários mais estudos sobre a suplementação de vitamina $D$ na prevenção de cancro, principalmente 
entre homens, indivíduos jovens e com défice de aporte alimentar de vitamina D. Seria importante avaliar, quer períodos mais longos de exposição aos suplementos de vitamina $\mathrm{D}$, quer doses mais elevadas destes.

\section{IMPLICAÇÕES PARA PRÁTICA}

1. A administração de suplementos de vitamina $D$ parece ter impacto na diminuição da mortalidade por cancro e

\section{REFERÊNCIAS}

1. Brouwer-Brolsma EM, Bischoff-Ferrari HA, Bouillon R, Feskens EJ, Gallagher CJ, Hypponen E, et al. Vitamin D: do we get enough? A discussion between vitamin $D$ experts in order to make a step towards the global (para todas as causas), mas o significado clínico deste benefício é incerto, estando apenas documentado em mulheres idosas.

2. Não há lugar a administração profiláctica de vitamina $D$ como medida de diminuição da mortalidade.

3. A administração combinada de vitamina $D$ e cálcio aumenta a incidência de casos de nefrolitíase.

harmonisation of dietary reference intakes for vitamin D across Europe Osteoporos Int. 2013;24:1567-77.

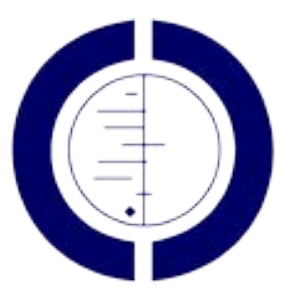

THE COCHRANE

COLLABORATION ${ }^{*}$ 


\section{Análise da Revisão Cochrane: Administração Suplementar de Vitamina D para Prevenção de Cancro em Adultos. Cochrane Database Syst Rev. 2014, 6:CD007469. Acta Med Port 2014:27:411-413}

Publicado pela Acta Médica Portuguesa, a Revista Científica da Ordem dos Médicos

Av. Almirante Gago Coutinho, 151

1749-084 Lisboa, Portugal.

Tel: +351218428 215

E-mail: submissao@actamedicaportuguesa.com

www.actamedicaportuguesa.com

ISSN:0870-399X | e-ISSN: 1646-0758

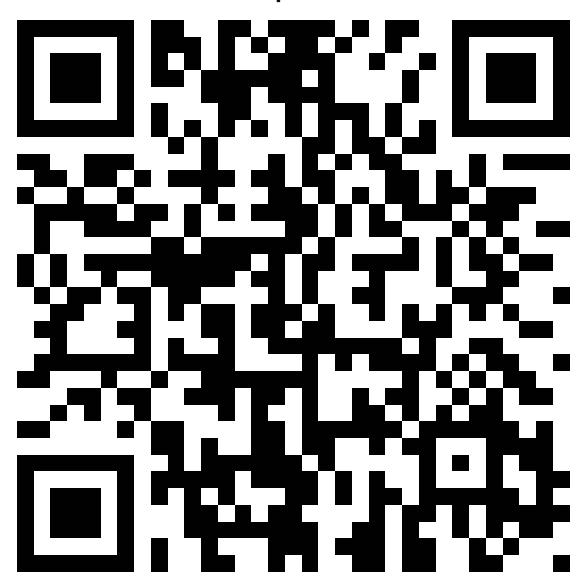

